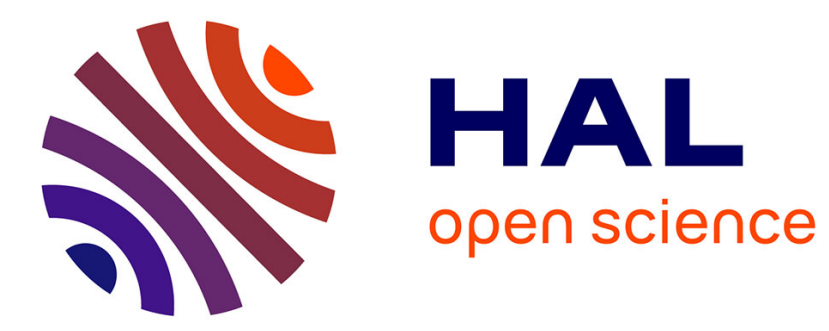

\title{
Comparaison des performances des capteurs héliothermiques plans
}

B. Drévillon, R. Vanderhaghen

\section{To cite this version:}

B. Drévillon, R. Vanderhaghen. Comparaison des performances des capteurs héliothermiques plans. Revue de Physique Appliquée, 1980, 15 (2), pp.131-138. 10.1051/rphysap:01980001502013100 . jpa00244707

\section{HAL Id: jpa-00244707 https://hal.science/jpa-00244707}

Submitted on 1 Jan 1980

HAL is a multi-disciplinary open access archive for the deposit and dissemination of scientific research documents, whether they are published or not. The documents may come from teaching and research institutions in France or abroad, or from public or private research centers.
L'archive ouverte pluridisciplinaire HAL, est destinée au dépôt et à la diffusion de documents scientifiques de niveau recherche, publiés ou non, émanant des établissements d'enseignement et de recherche français ou étrangers, des laboratoires publics ou privés. 


\title{
Comparaison des performances des capteurs héliothermiques plans
}

\author{
B. Drévillon et R. Vanderhaghen \\ LPNHE, Ecole Polytechnique, Route de Saclay, 91128 Palaiseau, France \\ (Reçu le 1er octobre 1979, révisé le 12 novembre 1979, accepté le 13 novembre 1979)
}

\begin{abstract}
Résumé. - Il est procédé à une comparaison systématique des performances des différents types de capteurs héliothermiques plans à l'aide des données météorologiques horaires disponibles à Trappes et à Carpentras. L'intégration des capteurs à un système de chauffage domestique ou à un système de production d'eau chaude sanitaire est étudiée. L'influence de plusieurs paramètres comme la température de fonctionnement, la surface de captation, l'orientation du capteur, l'inertie thermique et le volume de stockage est analysée. L'avantage des capteurs très performants (sélectif-anti-convectifs) est clairement établi. Ainsi, une surface de captation de $50 \mathrm{~m}^{2}$ en région parisienne permet d'obtenir, pour un volume de $225 \mathrm{~m}^{3}$ ayant un coefficient de perte volumique de $1,5 \mathrm{~W} / \mathrm{m}^{3}{ }^{\circ} \mathrm{C}$, des économies de chauffage de $45 \%$ à une température de fonctionnement de $70{ }^{\circ} \mathrm{C}$.

Abstract. - A systematic comparison of flat-plate collector efficiencies is performed, using hourly solar radiation measurements at Trappes and Carpentras. The integration to space heating or domestic hot water production systems is studied. The influence of some parameters as operation temperature, collection area, collector orientation, thermal inertia and thermal storage volume is considered. The interest of using collectors of high performance (selective-non-convective) is clearly established. A $50 \mathrm{~m}^{2}$ captation area of such collectors to heat a building of $225 \mathrm{~m}^{3}$ in volume and a volumic loss coefficient of $1.5 \mathrm{~W} / \mathrm{m}^{3}{ }^{\circ} \mathrm{C}$, enables $45 \%$ savings in the region of Paris at an operation temperature of $70^{\circ} \mathrm{C}$.
\end{abstract}

1. Introduction. - Les composantes directes et diffuses (sur le plan horizontal) de l'énergie solaire ainsi que la température sont mesurées, heure par heure, depuis plusieurs années à Trappes et à Carpentras. A partir de ces données, nous avons procédé à une comparaison systématique des performances des différents types de capteurs héliothermiques plans.

La méthode et les différentes hypothèses de calcul sont décrites dans la deuxième partie de l'article. Dans la troisième partie, le rendement des trois types de capteurs les plus répandus (noir ordinaire, sélectif, sélectif-anti-convectif) est étudié ainsi que l'influence de la température de captation et de l'inertie thermique. Dans la quatrième partie, nous analysons le fonctionnement des capteurs intégrés dans un système de chauffage domestique. Après avoir étudié l'influence du coefficient de pertes avant, nous analysons, pour les trois types de capteurs retenus, l'influence de la température de fonctionnement, de la surface de captation, de l'angle d'inclinaison et de l'azimut, du volume de stockage et de l'inertie thermique. L'intégration à un système de production d'eau chaude sanitaire est décrite dans la cinquième partie.
2. Méthode de calcul. - Dans ce qui suit, les plans de capteurs seront, sauf indication contraire, orientés vers le sud avec un angle de $60^{\circ}$ par rapport au plan horizontal. Nous avons donc été amenés à estimer l'énergie solaire diffuse sur ce plan à partir de sa mesure sur le plan horizontal (cf. annexe 1).

En tenant compte de la réflexion sur la face avant du verre, nous avons ainsi déterminé l'énergie reçue par le capteur, heure par heure [1, 2]. Nous nous sommes limités au cas du vitrage unique, supposé en équilibre thermique avec l'extérieur (cf. annexe 1).

Les pertes thermiques des faces avant et arrière du capteur ont été supposées proportionnelles aux écarts de température (cf. annexes 1 et 2). Les valeurs des coefficients de proportionnalité ont été choisies en accord avec les publications antérieures $[1,3]$.

L'inertie thermique du système de capteurs a été prise en compte en retranchant des apports énergétiques la part nécessaire à la mise en température du capteur, en partant de la température ambiante. La température de l'absorbeur ne différant que de quelques degrés de celle du fluide caloporteur, nous l'avons identifié à la température de fonctionnement 
du système $\left({ }^{1}\right)$. La capacité calorifique retenue est donc celle de l'ensemble capteur-fluide caloporteur.

Dans la quatrième partie nous analysons les performances des capteurs intégrés à un système de chauffage domestique. Nous indiquons alors dans chaque cas l'énergie fournie par les capteurs solaires, seules les pertes au niveau de l'absorbeur ont été prises en compte. Un stockage thermique de dimension variable, fonctionnant en permanence, a été supposé. Compte tenu de ce stockage, les apports énergétiques ont été plafonnés aux besoins de l'habitation. Nous avons procédé de même dans la cinquième partie (production d'eau chaude sanitaire).

Dans ce qui suit, il a toujours été procédé à une moyenne sur quatre années de fonctionnement (19711975).

3. Fonctionnement des capteurs. - Les performances mensuelles des trois types de capteurs héliothermiques plans considérés ont été calculées pour deux températures de fonctionnement $\left(40^{\circ} \mathrm{C}\right.$ et $\left.70^{\circ} \mathrm{C}\right)$. Nous avons caractérisé les différents capteurs par les

( $\left.{ }^{1}\right)$ Si l'on considère un capteur irrigué par un réseau de tuyaux transverses de $1 \mathrm{~cm}$ de diamètre, placés tous les $5 \mathrm{~cm}$, avec un débit linéaire de $1 \mathrm{~cm} / \mathrm{s}$. L'écart de température entre l'absorbeur et le fluide caloporteur est de $7{ }^{\circ} \mathrm{C}$ pour une irradiation utile de $500 \mathrm{~W} / \mathrm{m}^{2}$, l'écart de température entrée-sortie du caloporteur étant de $15^{\circ} \mathrm{C}$. Dans ce cas, la température de sortie est pratiquement égale à la température moyenne de l'absorbeur. Cependant, si l'écart de température entrée-sortie dans le capteur est grand $\left(40{ }^{\circ} \mathrm{C}\right)$, le rendement du système est sensiblement amélioré $(+5 \%$ pour le capteur II à Trappes, $+9 \%$ pour le capteur II à Carpentras pour $\left.T=70^{\circ} \mathrm{C}\right)$. valeurs du coefficient de pertes avant $k_{\mathrm{av}}$ et de l'absorptivité $\alpha$ suivants :

- sélectif-anti-convectif

$$
k_{\mathrm{av}}=1,3 \mathrm{~W} / \mathrm{m}^{2}{ }^{\circ} \mathrm{C}, \quad \alpha=0,9 \text { capteur I ; }
$$

- sélectif

$$
k_{\mathrm{av}}=4,5 \mathrm{~W} / \mathrm{m}^{2}{ }^{\circ} \mathrm{C}, \quad \alpha=0,9 \text { capteur II ; }
$$

- noir ordinaire

$$
k_{\mathrm{av}}=11 \mathrm{~W} / \mathrm{m}^{2}{ }^{\circ} \mathrm{C}, \quad \alpha=1 \text { capteur III . }
$$

En ce qui concerne le coefficient de perte arrière et la capacité calorifique, nous avons utilisé les valeurs habituelles $[1,3]$ de $1,5 \mathrm{~W} / \mathrm{m}^{2}{ }^{\circ} \mathrm{C}$ et de $5 \mathrm{~kJ} / \mathrm{m}^{2}{ }^{\circ} \mathrm{C}$.

Les résultats sont présentés sur la figure 1 . Nous constatons qu'à Trappes comme à Carpentras, l'introduction d'un système anti-convectif permet de doubler le rendement à une température d'utilisation de $70{ }^{\circ} \mathrm{C}$. Cet effet apparaît clairement sur la figure 2 qui représente la production énergétique des trois types de capteur en fonction de la température de fonctionnement, intégrée sur la période hivernale (octobre à mai). Ainsi, les températures auxquelles le rendement tombe au $3 / 4$ de sa valeur à $25^{\circ} \mathrm{C}$ sont respectivement pour les trois types de capteurs $32^{\circ} \mathrm{C}$, $37^{\circ} \mathrm{C}$ et $50^{\circ} \mathrm{C}$ à Trappes et $34^{\circ} \mathrm{C}, 43^{\circ} \mathrm{C}$ et $65^{\circ} \mathrm{C}$ à Carpentras.

L'influence de la capacité calorifique du capteur sur son rendement est indiquée dans le tableau I $a$. Nous constatons qu'une diminution de matière représente un gain de rendement inférieur à $10 \%$ (capteur noir à $70^{\circ} \mathrm{C}$ excepté). Par ailleurs, l'influence de l'inertie thermique est d'autant plus faible que le capteur est plus performant.

$$
\begin{gathered}
\mathrm{T}_{\mathrm{f}}=40^{\circ} \mathrm{C} \quad 70^{\circ} \mathrm{C} \\
\text { I } \Delta \\
\text { II } 0 \\
\text { III } 0
\end{gathered}
$$

ÉNERGIE UTILISABLE
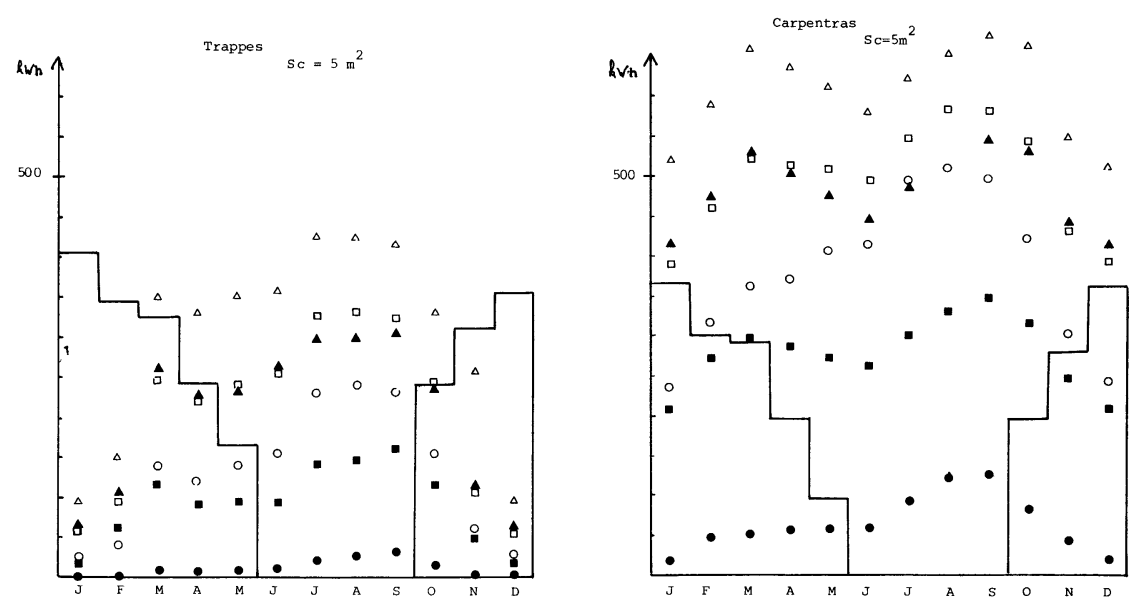

Fig. 1. - Energie recueillie par $5 \mathrm{~m}^{2}$ des différents capteurs, mois par mois. Le trait continu représente, à titre de comparaison, le dixième des besoins de chauffage de l'habitation de $225 \mathrm{~m}^{3}$ décrite plus loin.

[Monthly collected energy per $5 \mathrm{~m}^{2}$ collector. Full line represents, for comparison, the tenth of space heating needs of the $225 \mathrm{~m}^{3}$ building described after.] 


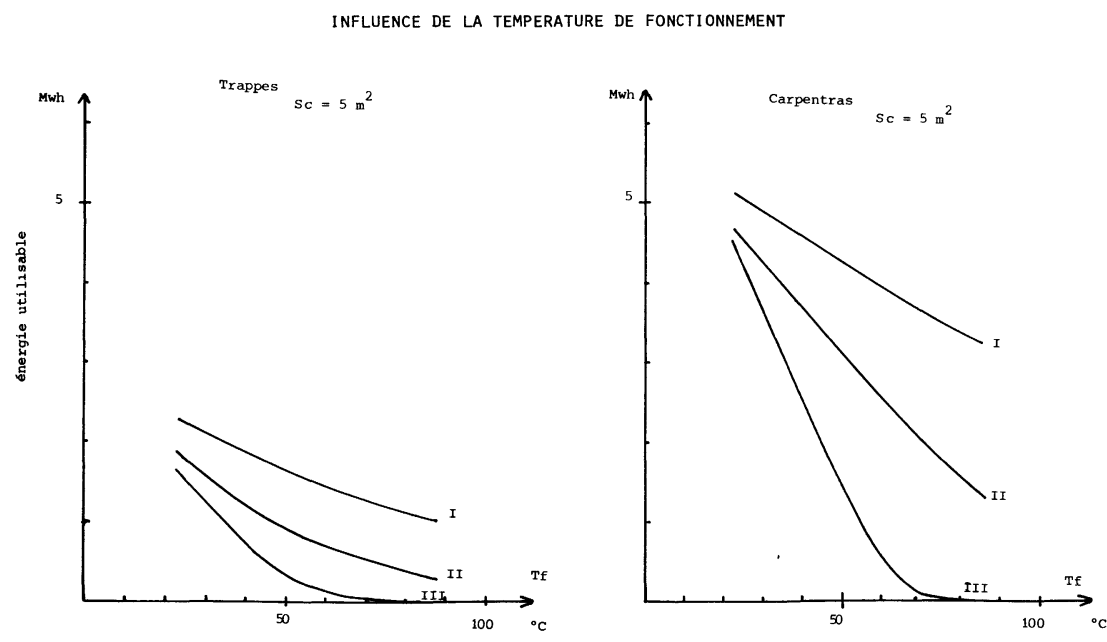

Fig. 2. - Influence de la température de fonctionnement sur l'énergie recueillie par les différents capteurs (d'octobre à mai).

[Operation temperature influence on the collected energy (from october to may).]

Tableau I. - Pertes de rendement dues à la capacité calorifique du capteur $\left(5 \mathrm{~kJ} / \mathrm{m}^{2}{ }^{\circ} \mathrm{C}\right)$ en proportion des apports énergétiques des capteurs (les résultats sont en pourcentage). Dans le b) les surfaces de captation sont de $50 \mathrm{~m}^{2}$ à Trappes et de $30 \mathrm{~m}^{2}$ à Carpentras.

[Efficiency losses due to the collector heat capacity $\left(5 \mathrm{~kJ} / \mathrm{m}^{2}{ }^{\circ} \mathrm{C}\right)$ in proportion of the collectors energy production (results are in percentage). In $b$ ) the collection area is $50 \mathrm{~m}^{2}$ at Trappes and $30 \mathrm{~m}^{2}$ at Carpentras.]

a) Capteur seul.

\begin{tabular}{|c|c|c|c|c|c|c|}
\hline Type de capteur & I & II & III & I & II & III \\
\hline- & - & - & - & - & - & - \\
\hline lieu & \multicolumn{3}{|c|}{ Trappes } & \multicolumn{3}{|c|}{ Carpentras } \\
\hline $40^{\circ} \mathrm{C}$ & 2,5 & 3,1 & 4,4 & 1,3 & 1,6 & \\
\hline $70^{\circ} \mathrm{C}$ & 5,6 & 9,9 & 31,0 & 2,9 & 5,1 & \\
\hline
\end{tabular}

b) Intégration au chauffage domestique.

Type de capteur

lieu

$40^{\circ} \mathrm{C}$

$\begin{array}{ccr}\text { I } & \text { II } & \text { III } \\ - & - & - \\ & \text { Trappes } & \\ 2,0 & 2,7 & 4,6 \\ 4,9 & 10,5 & 41,2\end{array}$

$\begin{array}{lrr}\text { I } & \text { II } & \text { III } \\ - & - & - \\ \text { Carpentras } & \\ 0,6 & 1,0 & 1,9 \\ 4,5 & 1,6 & 25,2\end{array}$

4. Intégration dans un système de chauffage. L'intégration des capteurs à un système de chauffage domestique, dans les conditions décrites au chapitre 2 , modifie les résultats précédents. A titre d'exemple, nous avons considéré le cas d'une maison de $225 \mathrm{~m}^{3}$ ayant un coefficient de déperdition volumique $G=1,5 \mathrm{~W} / \mathrm{m}^{3}{ }^{\circ} \mathrm{C}$ chauffée en permanence d'octobre à mai à $19^{\circ} \mathrm{C}$.

Nous avons supposé l'existence d'un stockage sans perte de $150 \mathrm{kWh}$ (stockage journalier) fonctionnant en permanence. Il peut être réalisé soit par un corps changeant d'état à la température de fonctionnement considéré ou bien par l'utilisation de la chaleur sensible d'un corps stratifié thermiquement.

Les autres caractéristiques des capteurs (capacité calorifique, pertes thermiques) sont celles indiquées au chapitre 3 . Les surfaces de captation choisies sont de $50 \mathrm{~m}^{2}$ à Trappes et $30 \mathrm{~m}^{2}$ à Carpentras.

Nous allons étudier successivement l'influence de chaque paramètre.

4.1 Coefficient De Perte avant. - Les performances de tous les types possibles de capteurs héliothermiques plans sont indiquées sur la figure 3 . Nous avons fait varier le coefficient de perte avant $k_{\mathrm{av}}$ de $0,8 \mathrm{~W} / \mathrm{m}^{2}{ }^{\circ} \mathrm{C}$ (capteur idéal) à $11 \mathrm{~W} / \mathrm{m}^{2}{ }^{\circ} \mathrm{C}$. Nous constatons que des économies annuelles de chauffage supérieures ou de l'ordre de $50 \%$ à des températures de fonctionnement supérieures à $40^{\circ} \mathrm{C}$ nécessitent, en région parisienne, des capteurs ayant des coefficients $k_{\text {av }}$ inférieurs à $2 \mathrm{~W} / \mathrm{m}^{2}{ }^{\circ} \mathrm{C}$ (capteurs sélectifsanti-convectifs).

4.2 TeMpérature DE FONCTIONNEMENT. - L'influence de la température de fonctionnement pour les trois types de capteurs considérés est donnée par la figure 4. Les températures auxquelles l'apport énergétique tombe aux $3 / 4$ de sa valeur à $25^{\circ} \mathrm{C}$ sont respectivement pour les trois capteurs $33^{\circ} \mathrm{C}, 43^{\circ} \mathrm{C}$ et $63^{\circ} \mathrm{C}$ à Trappes et $40^{\circ}, 59^{\circ} \mathrm{C}$ et $108^{\circ} \mathrm{C}$ à Carpentras. Des capteurs très performants peuvent donc fonctionner à des températures élevées sans dégrader notablement leur apport énergétique, ce qui permet leur incorporation aisée dans certaines systèmes de climatisation [4].

4.3 Surface de captation. - L'influence de la surface de captation est illustrée par la figure 5 . Le plafonnement aux besoins et le stockage limité rendent rapidement inefficace l'augmentation de la surface de captation. Ainsi, nous voyons que la surface dont 


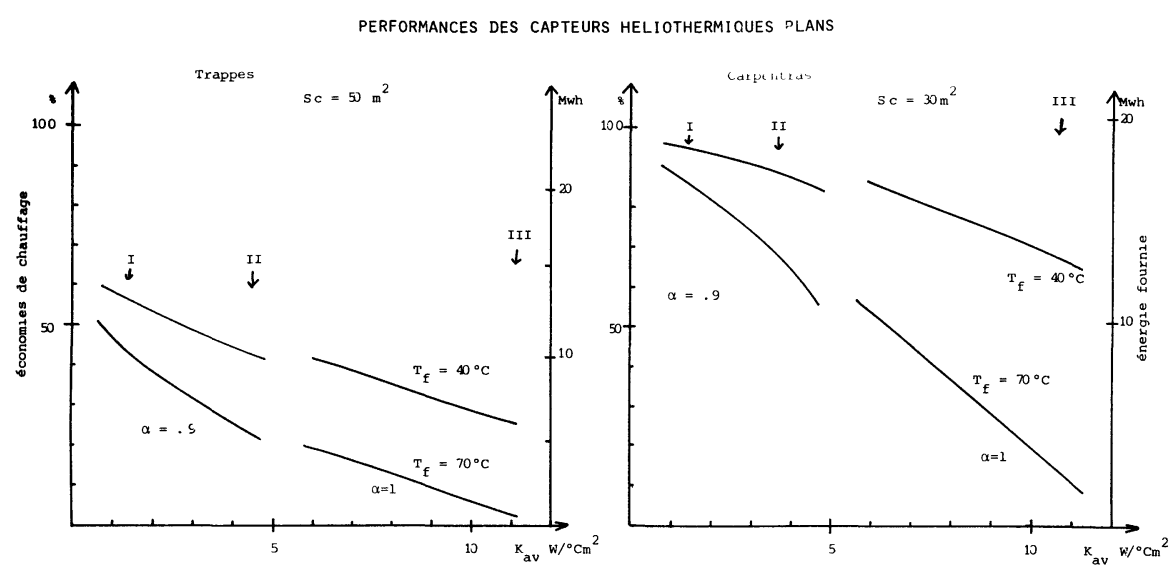

Fig. 3. - Comparaison des performances des différents types de capteurs héliothermiques plans intégrés dans un système de chauffage domestique.

[Performance comparison of different kinds of flat-plate collectors integrated to a space heating system.]

INFLUENCE DE LA TEMPERATURE DE FONCTIONNEMENT
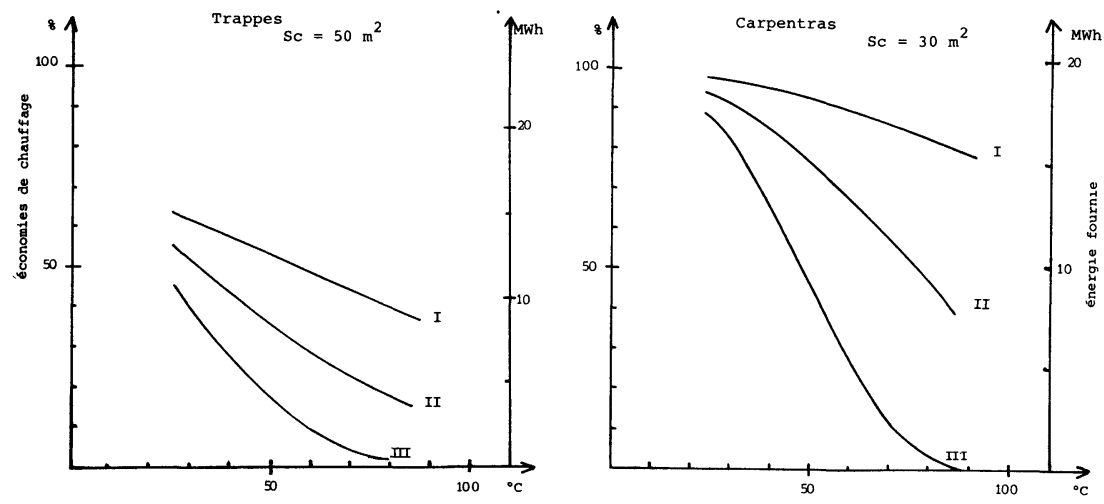

Fig. 4. - Influence de la température de fonctionnement sur les performances des trois types de capteurs intégrés à un système de chauffage.

[Operation temperature influence on performances of the three collectors integrated to a space heating system.]

INFLUENCE DE LA SURFACE DE CAPTATION

$$
\begin{aligned}
\mathrm{T}_{f} & =40^{\circ} \mathrm{C} \\
---\mathrm{T}_{\mathrm{f}}=70^{\circ} \mathrm{C} &
\end{aligned}
$$
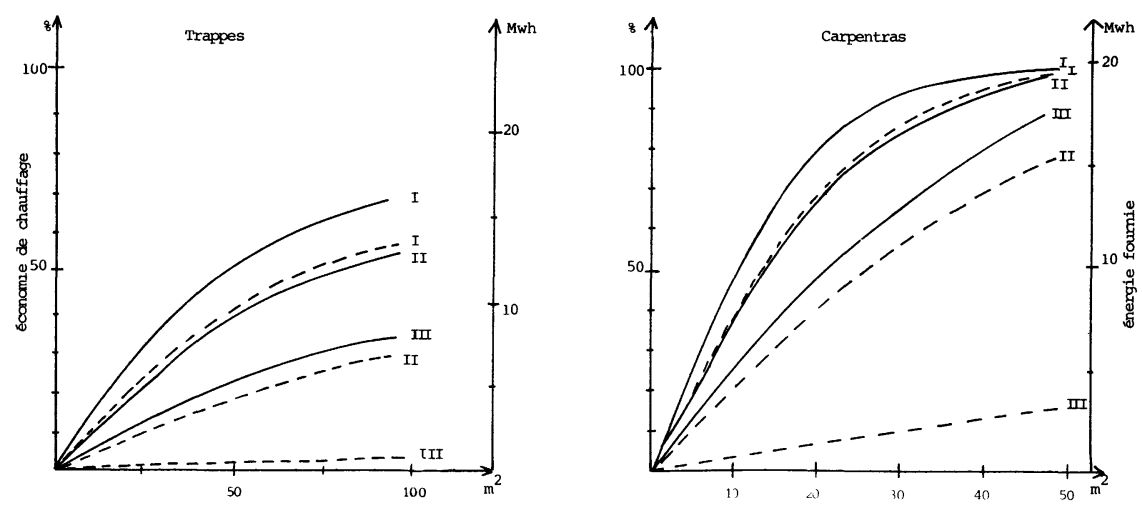

Fig. 5. - Influence de la surface de captation sur les performances des trois types de capteurs intégrés à un système de chauffage. [Collection area influence on performances of the three collectors integrated to a space heating system.] 
l'apport marginal est $50 \%$ de l'apport initial (le $\mathrm{m}^{2}$ supplémentaire produit la moitié de ce que produit le premier $\mathrm{m}^{2}$ ) est, à $70^{\circ} \mathrm{C}$, de $50 \mathrm{~m}^{2}$ à Trappes et $26 \mathrm{~m}^{2}$ à Carpentras pour le capteur I. Cette surface passe à $92 \mathrm{~m}^{2}$ à Trappes et $46 \mathrm{~m}^{2}$ à Carpentras pour le capteur II. Les apports de chauffage sont alors de $45 \%$ à Trappes et $79 \%$ à Carpentras pour le capteur le plus performant. Ceci représente une surface de capteur de $47 \mathrm{~cm}^{2}$ à Trappes et de $17 \mathrm{~cm}^{2}$ à Carpentras (à $70^{\circ} \mathrm{C}$ ) par $\mathrm{kWh}$ fourni. Ce dernier chiffre est à comparer avec les $36 \mathrm{~cm}^{2}$ de la maison solaire d'Aramon [5].

4.4 ANGLE D'INClinaison ET AZIMUT. - L'influence de l'angle d'inclinaison du capteur par rapport au plan horizontal ainsi que l'effet de son orientation par rapport au sud sont indiqués par la figure 6 .

L'effet de l'inclinaison diminue avec les performances du capteur. Les capteurs noirs ordinaires étant peu performants l'hiver, leur inclinaison optimum se situe aux alentours de $45^{\circ}$.

Nous constatons également sur la figure 6 que les azimuts qui correspondent à un rendement équivalent à $90 \%$ de celui du sud sont d'environ $\pm 35^{\circ}$ à Trappes comme à Carpentras.

4.5 INERTIE THERMIQUE. - Le tableau I indique les diminutions de rendement causées par la capacité calorifique $\left(5 \mathrm{~kJ} / \mathrm{m}^{2}{ }^{\circ} \mathrm{C}\right)$ des capteurs. On constate, en comparant avec les résultats équivalents du chapitre 3, que le plafonnement aux besoins limite les pertes de rendement.

4.6 Volume DE STOCKAGE. - La figure 7 représente l'influence du volume de stockage. La nécessité du stockage est d'autant plus grande que la performance du capteur est mauvaise. L'optimum se situe

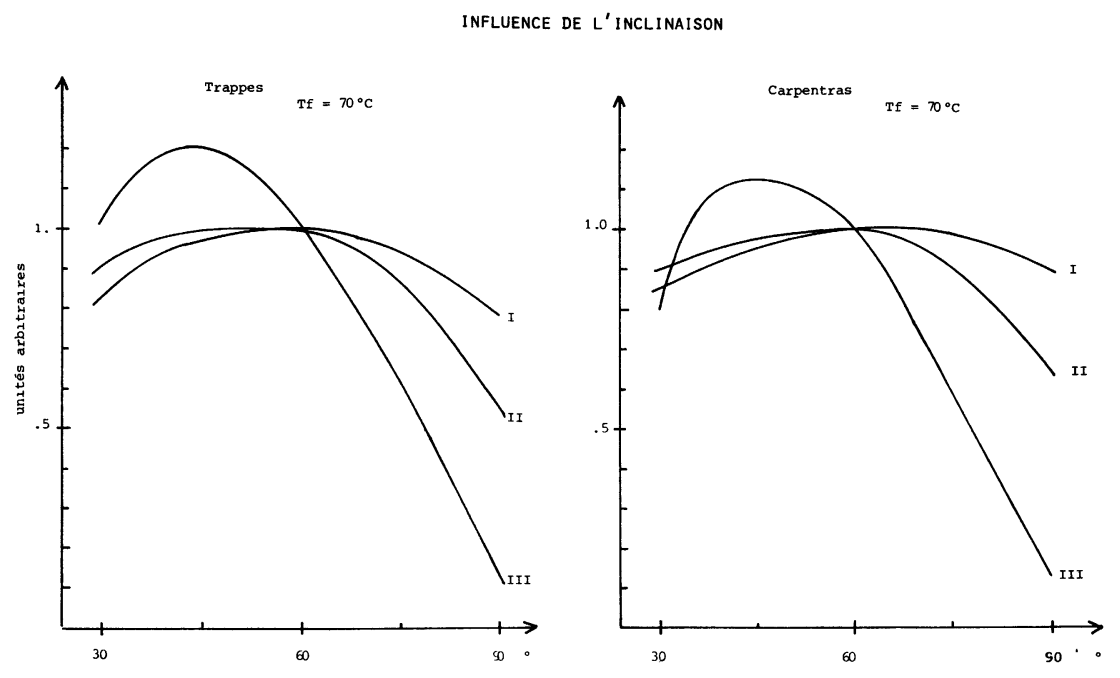

a)

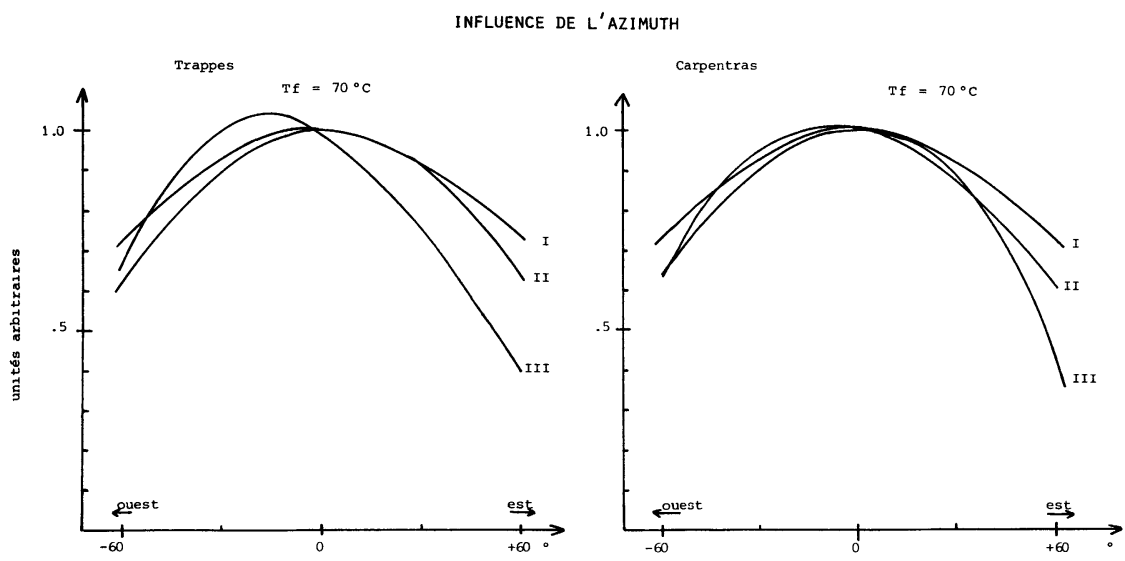

b)

Fig. 6. - Influences de l'inclinaison du capteur par rapport au plan horizontal et de l'azimut (par rapport au sud) sur les performances des trois types de capteurs intégrés à un système de chauffage $\left(T_{\mathrm{f}}=70^{\circ} \mathrm{C}\right)$. Les courbes ont été normalisées aux valeurs correspondant à une orientation sud et une inclinaison de $60^{\circ}$.

[Collector tilt (related to the horizontal plane) and azimuth (related to the south) on performances of three collectors integrated to a space heating system.] 


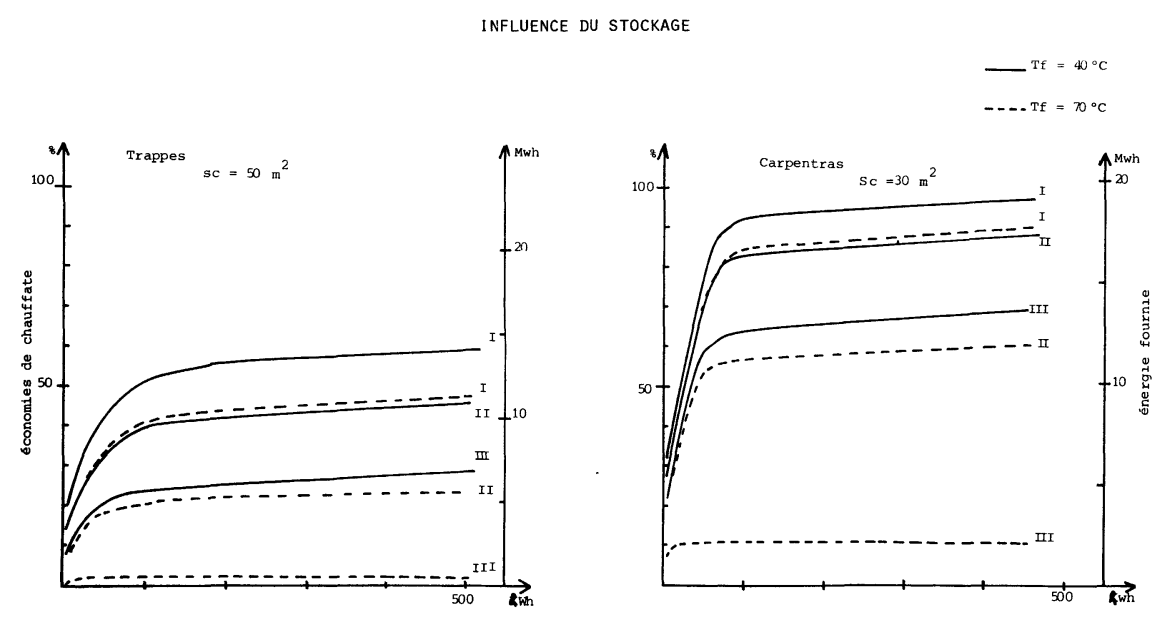

Fig. 7. - Influence de la dimension du stockage sur les performances des trois types de capteurs intégrés à un système de chauffage.

[Thermal storage influence on performances of the three collectors integrated to a space heating system.]

aux alentours de $200 \mathrm{kWh}$ à Trappes et de $100 \mathrm{kWh}$ à Carpentras, ce qui correspond, respectivement par $\mathrm{m}^{2}$ de capteur, à 1351 et $110 \mathrm{l}$. Ce résultat est comparable aux 1001 de $P$. Chouard et al. [2].

D'une manière générale, la figure 7 fait apparaître deux régimes. D'abord, une forte croissance qui reflète l'influence du stockage sur les besoins nocturnes et les variations météorologiques à court terme. Puis, une faible croissance qui traduit les effets à plus long terme.

5. Intégration à un système d'eau chaude sanitaire. Nous avons considéré, à titre d'exemple, le cas d'une consommation quotidienne de $10 \mathrm{kWh}\left(\right.$ à $\left.60^{\circ} \mathrm{C}\right)$ constante sur toute l'année, avec un stockage équivalent aux besoins d'une journée. Les apports énergétiques des trois types de capteurs sont présentés sur la figure 8 en fonction de la surface de captation $\left({ }^{2}\right)$. $\mathrm{La}$ surface nécessaire à la fourniture de $60 \%$ des besoins annuels est respectivement, pour les capteurs I et II, 4 et $9 \mathrm{~m}^{2}$ à Trappes et 1,5 et $2,0 \mathrm{~m}^{2}$ à Carpentras.

6. Conclusion. - Cette étude systématique des performances des différents types de capteurs héliothermiques plans, à l'aide de données météorologiques horaires, montre clairement l'importance de disposer de capteurs à hautes performances, spécialement en région parisienne. Il s'agit de capteurs ayant un coeffi-

(2) Pour ce calcul, étant donné les grands écarts de température, nous avons tenu compte de la différence de température du capteur entre l'entrée et la sortie $\left(T_{\text {entrée }}=15^{\circ} \mathrm{C}\right)$ et de la différence de température entre capteur et le caloporteur $\left(7^{\circ} \mathrm{C}\right.$ pour $500 \mathrm{~W} / \mathrm{m}^{2}$ utiles $)$.

PRODUCTION D'EAU CHAUDE SANITAIRE
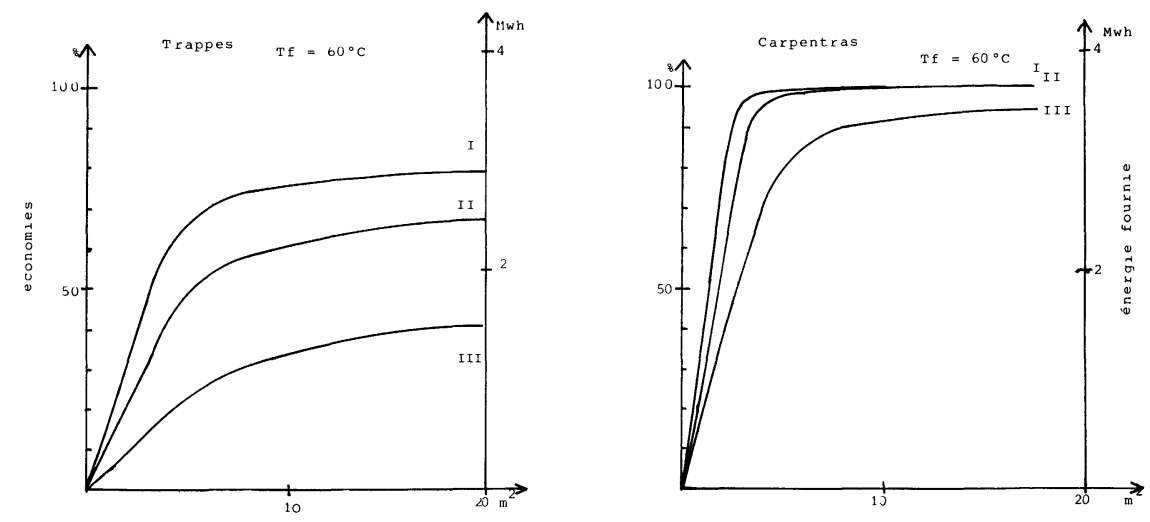

Fig. 8. - Influence de la surface de captation sur les performances des trois types de capteurs intégrés à un système de production d'eau chaude sanitaire.

[Collection area influence on performances of the three collectors integrated to a domestic hot water production system.] 
cient de pertes avant de l'ordre de $1,5 \mathrm{~W} / \mathrm{m}^{2}{ }^{\circ} \mathrm{C}$, ou moins. Cela impose que le capteur solaire soit revêtu d'un absorbant sélectif et dispose d'un système anticonvectif.

Seuls, ces capteurs peuvent fonctionner à des températures relativement élevées sans dégrader leurs propriétés énergétiques. Ainsi, si l'on considère un volume à chauffer de $225 \mathrm{~m}^{3}$, ils permettent un apport $(a ̀) 60^{\circ} \mathrm{C}$ ) énergétique de l'ordre de $50 \%$ des besoins pour une surface de captation de $50 \mathrm{~m}^{2}$ à Trappes, et de $90 \%$ environ pour $30 \mathrm{~m}^{2}$ à Carpentras. Dans cette gamme de température, la nature anti-convective du capteur permet de diminuer de moitié la surface de captation nécessaire.

\section{ANNEXES}

1. Energie solaire absorbée. - Définissons les notations suivantes :
$\lambda \quad$ latitude du lieu considéré.
$\delta \quad$ déclinaison du soleil.
$\omega$ angle horaire (zéro à midi).
$\theta_{\mathrm{p}}$ inclinaison du capteur par rapport au plan horizontal
$\psi \quad$ azimut du capteur (zéro au sud, positif à l'est).
$\theta_{\mathrm{i}} \quad$ angle d'incidence sur le verre.
$\theta_{\mathrm{r}}$ angle de réfraction dans le verre.
$I_{\mathrm{D}}$ intensité directe du rayonnement solaire (sur une surface normale). $I_{\mathrm{d}} \quad$ intensité diffuse du rayonnement solaire sur
une surface horizontale.
$I_{\mathrm{t}}$ intensité totale du rayonnement solaire sur une surface horizontale.
$\theta_{\mathrm{s}}$ angle d'incidence du rayonnement direct sur le plan horizontal.

$\rho \quad$ coefficient de réflexion du sol (on a supposé $\rho=0,2)$.

$k_{\text {av }}$ coefficient de perte avant.

$k_{\mathrm{ar}}$ coefficient de perte arrière.

$T_{\mathrm{f}} \quad$ température capteur.

$T_{\mathrm{ex}}$ température extérieure.

$T_{\text {int }}$ température intérieure (ici $19{ }^{\circ} \mathrm{C}$ ).

$\alpha \quad$ coefficient d'absorption du capteur.

$S_{\mathrm{c}}$ surface de captation.

L'angle d'incidence $\theta_{c}$ du rayonnement direct sur le capteur est donnée par [1]

$$
\begin{aligned}
\cos \theta_{\mathrm{c}}=\sin \delta \sin \lambda \cos \theta_{\mathrm{p}}-\sin \delta \cos \lambda \sin \theta_{\mathbf{p}} \cos \psi+ \\
+\cos \delta \cos \lambda \cos \theta_{\mathrm{p}} \cos \omega \\
+\cos \delta \sin \lambda \sin \theta_{\mathrm{p}} \cos \psi \cos \omega \\
+\cos \delta \sin \theta_{\mathrm{p}} \sin \psi \sin \omega .
\end{aligned}
$$

Les coefficients de réflexion $R\left(\theta_{\mathrm{i}}\right)$ et de transmission $T\left(\theta_{\mathrm{i}}\right)$ de la vitre avant sont :

$$
\begin{aligned}
R\left(\theta_{\mathrm{i}}\right)=1 & -T\left(\theta_{\mathrm{i}}\right)= \\
= & \frac{1}{2}\left[\left(\frac{\sin \left(\theta_{\mathrm{r}}-\theta_{\mathrm{i}}\right)}{\sin \left(\theta_{\mathrm{r}}+\theta_{\mathrm{i}}\right)}\right)^{2}+\left(\frac{\operatorname{tg}\left(\theta_{\mathrm{r}}-\theta_{\mathrm{i}}\right)}{\operatorname{tg}\left(\theta_{\mathrm{r}}+\theta_{\mathrm{i}}\right)}\right)^{2}\right] .
\end{aligned}
$$

Les composants $I_{\mathrm{D}}, I_{\mathrm{d}}$ et $I_{\mathrm{t}}$ du rayonnement solaire sont mesurés, heure par heure, à Trappes et à Carpentras. Nous avons supposé que le rayonnement diffus est constitué par deux composantes : une partie $\gamma$ directive, centrée sur la direction du soleil et une partie $(1-\gamma)$ isotrope. Nous avons supposé, de plus, que $\gamma$ était proportionnel à la part du rayonnement direct (sur le total) mesurée sur une surface normale à la direction du soleil :

$$
\gamma=\beta \frac{I_{\mathrm{D}}}{I_{\mathrm{D}}+I_{\mathrm{d}} \frac{\cos \theta_{\mathrm{c}}}{\cos \theta_{\mathrm{s}}}}
$$

Nous avons déduit la valeur de $\beta$ de mesures expérimentales du rayonnement solaire sur différentes surfaces $[6,7]$, elles donnent $\beta=0,8$. Si l'on pose $\beta=0$ (rayonnement diffus totalement isotrope) l'énergie absorbée est diminuée d'environ $10 \%$.

On en déduit alors, en tenant compte de la réflexion par le sol :

Rayonnement direct :

$$
I_{\mathrm{D}}^{R}=I_{\mathrm{D}} \cos \theta_{\mathrm{c}} T\left(\theta_{\mathrm{c}}\right),
$$

Rayonnement diffus directif :

$$
I_{\mathrm{DD}}^{R}=\gamma I_{\mathrm{d}} \cos \theta_{\mathrm{c}} T\left(\theta_{\mathrm{c}}\right) .
$$

Rayonnement diffus isotrope :

$$
I_{\mathrm{DI}}^{R}=(1-\gamma) I_{\mathrm{d}}\left(\frac{1-\sin \theta_{\mathrm{p}}}{2}\right) T\left(\frac{\theta_{\mathrm{p}}}{2}\right)
$$

Rayonnement diffus par le sol :

$$
I_{\mathrm{DS}}^{R}=\rho I_{\mathrm{t}} \frac{\sin \theta_{\mathrm{p}}}{2} T\left(\frac{\pi / 2-\theta_{\mathrm{p}}}{2}\right) .
$$

L'énergie absorbée, heure par heure, est alors :

$$
E_{\mathrm{abs}}=\alpha S_{\mathrm{c}}\left(I_{\mathrm{D}}^{R}+I_{\mathrm{DD}}^{R}+I_{\mathrm{DI}}^{R}+I_{\mathrm{DS}}^{R}\right) .
$$

Compte tenu des pertes, l'énergie utile est finalement :

$$
E_{\mathrm{u}}=E_{\mathrm{abs}}-\left[k_{\mathrm{av}}\left(T_{\mathrm{f}}-T_{\mathrm{ex}}\right)+k_{\mathrm{ar}}\left(T_{\mathrm{f}}-T_{\mathrm{int}}\right)\right] .
$$

2. Définition de quelques paramètres. - 2.1 PERTES ARRIÈRE. - On a choisi $k_{\mathrm{ar}}=1,5 \mathrm{~W} / \mathrm{m}^{2}{ }^{\circ} \mathrm{C}(5 \mathrm{~cm}$ de laine de verre donnent $k_{\text {ar }} \sim 0,7 \mathrm{~W} / \mathrm{m}^{2}{ }^{\circ} \mathrm{C}$ ). 
2.2 Pertes avant. - Si $\varepsilon$ désigne l'émissivité du capteur, les pertes par rayonnement entre 10 et $60^{\circ}$ sont de la forme $6,7 \varepsilon \mathrm{W} / \mathrm{m}^{2} \mathrm{~K}, \varepsilon$ variant de 0,1 (capteur sélectif) à 1 (noir ordinaire) [1,3]. Pour les pertes par convection et par conduction, on a utilisé respectivement $2,9 \mathrm{~W} / \mathrm{m}^{2}{ }^{\circ} \mathrm{C}$ et $0,6 \mathrm{~W} / \mathrm{m}^{2}{ }^{\circ} \mathrm{C}$, ce qui correspond à une distance vitre-absorbeur de $4 \mathrm{~cm}$ avec un angle de $60^{\circ}[1]$.

2.3 INERTIE THERMIQUE. - La valeur choisie $\left(5 \mathrm{~kJ} / \mathrm{m}^{2}{ }^{\circ} \mathrm{C}\right)$ de la capacité calorifique correspond à une plaque de $1 \mathrm{~mm}$ de cuivre d'épaisseur et un volume d'eau de $0,331 / \mathrm{m}^{2}$.

2.4 Stockage. - Les besoins moyens de chauffage sont en janvier à Trappes, pour la maison considérée, de $125 \mathrm{kWh} / \mathrm{jour}$. Un stockage de $100 \mathrm{kWh}$, avec une différence de température de $30^{\circ} \mathrm{C}$, représente un volume de $2,9 \mathrm{~m}^{3}$ d'eau.

\section{Bibliographie}

[1] Duffie, J. A., Beckman, W. A., Solar Energy thermal process. (John Wiley and Sons New York) 1974.

[2] Chouard, P., Michel, H., Simon, M. F., Bilan thermique d'une maison solaire (Eyrolles, Paris) 1977.

[3] Devin, B., Analyse physique et expérimentale des capteurs plans en régime statique CEA/CPES/RT-77-033.

[4] Hinotani, K., Kanatani, M. and Osumi, M., An evacuated glass tube solar collector and its application to a solar cooling, heating and hot water supply system for the hospital in Kinki university. Sol. Energy 22 (1979) 535.
[5] Chouard, P., Une année d'expérience en Aramon : les bilans prévisionnels face aux résultats de mesures. EDF/HE $112 \mathrm{~W} 1228$.

[6] Heywood, H., The computation of solar radiation intensities. Sol. Energy 10 (1966) 51.

[7] Tomps, R. C., Coulson, K. L., Solar radiation incident upper slopes of different orientations. Sol. Energy 19 (1977) 179. 